\title{
Long non-coding RNA T cell factor 7 is associated with increased disease risk and poor prognosis, and promotes cell proliferation, attenuates cell apoptosis and miR-200c expression in multiple myeloma
}

\author{
TIANLING DING ${ }^{1}$, RUOYU DENG ${ }^{2}$ and TING HUANG ${ }^{3}$ \\ ${ }^{1}$ Department of Hematology, Huashan Hospital Affiliated to Fudan University, Shanghai 200040; \\ ${ }^{2}$ Shanghai Qeejen Bio-tech Institution, Shanghai 200434; ${ }^{3}$ Department of Oncology, The Central Hospital of Wuhan, \\ Tongji Medical College, Huazhong University of Science and Technology, Wuhan, Hubei 430014, P.R. China
}

Received August 20,2019; Accepted April 20, 2020

DOI: $10.3892 / \mathrm{ol} .2020 .12390$

\begin{abstract}
The aim of the present study was to investigate the association of long non-coding RNA T cell factor 7 (lncRNA TCF7) with disease risk, prognosis and its cellular function in multiple myeloma (MM). A total of 132 de novo symptomatic patients with MM and 50 controls were enrolled. Plasma cells from patients with MM and controls were separated from bone marrow samples to detect lncRNA TCF7 expression using reverse transcription-quantitative PCR. In addition, treatment responses, event-free survival (EFS) and overall survival (OS) were measured. The effects of 1ncRNA TCF7 on proliferation, apoptosis and microRNA-200c (miR-200c) expression were assessed by gain- and loss-of-function experiments in RPMI-8226 and U-266 cells. The results demonstrated that lncRNA TCF7 expression was upregulated in patients with MM compared with controls, and the receiver operating characteristic curve revealed that lncRNA TCF7 could distinguish patients with
\end{abstract}

Correspondence to: Professor Ting Huang, Department of Oncology, The Central Hospital of Wuhan, Tongji Medical College, Huazhong University of Science and Technology, 26 Shengli, Wuhan, Hubei 430014, P.R. China

E-mail: lu34472056@163.com

Abbreviations: MM, multiple myeloma; lncRNAs, long non-coding RNAs; lncRNA TCF7, lncRNA T-cell factor 7; Hb, hemoglobin; Scr, serum creatinine; ALB, albumin; LDH, lactate dehydrogenase; $\beta 2-\mathrm{MG}, \beta 2$-microglobulin; ISS, International Staging System; RT-qPCR, reverse transcription-quantitative PCR; NCCN, National Comprehensive Cancer Network; CR, complete response; VGPR, very good partial response; PR, partial response; ORR, overall response rate; EFS, event-free survival; OS, overall survival; miR-200c, microRNA-200c; ROC, receiver operating characteristic; AUC, area under the curve

Key words: disease risk, lncRNA TCF7, MM, prognosis, tumorigenesis
MM from controls with an area under the curve of 0.793 (95\% CI, 0.725-0.861). In patients with MM, high lncRNA TCF7 expression was associated with higher $\beta 2$-microglobulin, more advanced International Staging System stage and increased $t(14 ; 16)$ mutations. Furthermore, it was demonstrated that lncRNA TCF7 was downregulated in patients with complete response (CR) compared with patients without CR. Furthermore, high lncRNA TCF7 expression predicted worse EFS and OS. IncRNA TCF7 also promoted cell proliferation, whereas it reduced cell apoptosis and miR-200c expression in RPMI-8226 and U-266 cells. In conclusion, the present results suggested that lncRNA TCF7 may be used as a potential biomarker and as a treatment target for MM.

\section{Introduction}

Multiple myeloma (MM), a B-cell malignancy, is characterized by the accumulation of malignant plasma cells within the bone marrow, and is the second most common hematological malignancy with an age-standardized incidence rate of 2.1 per 100,000 individuals worldwide in $2016(1,2)$. The introduction of autologous stem cell transplantation, proteasome inhibitors and immunomodulatory agents has increased the 5-year survival rate from 26.5 to $44.9 \%$ in patients with MM $(3,4)$. However, a proportion of patients with MM relapse or become refractory to these treatments (5). Therefore, it is important to identify novel treatment targets for improving prognosis in patients with MM.

Following the development and wide application of high-throughput sequencing and bioinformatics analyses in clinical research, the role of non-coding RNAs in the pathogenesis of MM has been investigated (6). As a class of non-coding RNAs, long non-coding RNAs (lncRNAs) are defined as non-coding transcripts $>200$ nucleotides long with 5 ' and $3^{\prime}$ ends (7). IncRNAs regulate gene transcription and mRNA translation by interacting with other components including proteins, RNA and DNA (7). Previous studies have shown that some lncRNAs, such as lncRNA metastasis associated lung adenocarcinoma transcript 1 (MALAT1) and lncRNA H19, are involved in the development and progression 
of MM $(3,8)$. lncRNA T-cell factor 7 (lncRNA TCF7) has been widely studied regarding its role in facilitating proliferation and self-renewal of cancer cells in solid tumors, including colorectal cancer and glioma (9-11). Moreover, lncRNA TCF7 has been reported to regulate TCF7 expression by recruiting the SWItch/Sucrose Non-Fermentable complex to the promoter of TCF7, resulting in activation of the Wnt signaling pathway, which is a key oncogene pathway implicated in the development and progression of MM $(12,13)$. Therefore, it was hypothesized that lncRNA TCF7 may be involved in the pathogenesis of MM. Thus, the aim of the present study was to investigate the association of lncRNA TCF7 with disease risk, prognosis and its cellular function in MM.

\section{Patients and methods}

Participants. Initially, 237 patients with MM were enrolled, and 19 patients were excluded from the study due to refusal to attend a pre-screening procedure. Among 218 eligible patients, 86 patients were excluded (64 patients did not meet inclusion criteria or met exclusion criteria, and 22 patients refused to sign informed consent forms; Fig. 1). In total, 132 newly diagnosed patients with MM (age range, 42-76 years; 82 males and 50 females) and 50 controls (age range, 45-65 years; 31 males and 19 females) were recruited from The Central Hospital of Wuhan (Wuhan, China) between January 2016 and December 2018. The screening criteria of patients with MM were as follows: i) Diagnosed as de novo symptomatic MM according to International Myeloma Working Group Recommendations for Global Myeloma Care (2014) (14); ii) aged $>18$ years old; iii) without concurrence of other tumors or other hematopoietic malignancies; iv) no serious infection (e.g., human immunodeficiency virus); v) no history of chemotherapy, radiotherapy or other systematic treatments; vi) no pregnancy; and vii) willing to provide written informed consent. The control individuals consisted of bone marrow donors with anemia and patients with non-hematopoietic malignancy who underwent bone marrow puncture at the hospital due to suspicion of immune thrombocytopenic purpura or iron deficiency. All the control individuals were $>18$ years old and had no history of malignancies or severe infection. This study was conducted in accordance with ethical standards and was approved by the Institutional Review Board of The Central Hospital of Wuhan, Tongji Medical College, Huazhong University of Science and Technology. If participants presented with severe symptoms, their families/legal guardians were legally responsible for acquiring the consent information, making the decision and signing the consent form. All participants or their guardians provided written informed consent.

Baseline data and bone marrow sample collection. Baseline data of patients with MM, including age, sex, immunoglobulin subtype, bone lesion, hemoglobin ( $\mathrm{Hb}$ ), calcium, serum creatinine (Scr), albumin (ALB), $\beta 2$-microglobulin ( $\beta 2-\mathrm{MG}$ ), lactate dehydrogenase (LDH), Durie-Salmon stage, International Staging System (ISS) stage and cytogenetics abnormality were assessed. Cytogenetics abnormalities were determined by fluorescence in situ hybridization (FISH). Prior to the detection of cytogenetics abnormalities by FISH, plasma cells were purified from bone marrow samples of all patients using CD138-conjugated magnetic beads according to the manufacturer's protocol (Miltenyi Biotec $\mathrm{GmbH}$ ). Subsequently, FISH was performed to identify $t(4 ; 14), t(14 ; 16)$ and Del (17p). IGH/FGFR3, IGH/MAF and P53 commercial probes (purchased from Beijing GP Medical Technologies Ltd.) were used to detect t $(4 ; 14), \mathrm{t}(14 ; 16)$ and Del $(17 \mathrm{p})$, respectively. Fluorescence images were captured using an epifluorescence microscope (magnification, x100; Leica DRMA2; Leica Microsystems $\mathrm{GmbH}$ ) equipped with a charge-coupled device camera (Andor Technology; Oxford Instruments) and appropriate filters. A total of 200 nuclei were scored for each probe. The cut-off values for positive results for each probe in FISH were estimated according to the recommendations of the European Myeloma Network FISH workshops as follows: $10 \%$ for fusion and $20 \%$ for numerical abnormalities (15). The Durie-Salmon stage was assessed in accordance with the Durie-Salmon staging system (16), and ISS stage was evaluated referring to the ISS staging system (17).

Bone marrow samples from patients with MM were collected before treatment initiation, and bone marrow samples from controls were collected on enrollment. After collection, the bone marrow samples were separated to obtain mononuclear cells by density gradient centrifugation at $2,000 \mathrm{x}$ g for $10 \mathrm{~min}$ at room temperature, and CD138-positive plasma cells were isolated from mononuclear cells using CD138-coated magnetic beads (Miltenyi Biotec $\mathrm{GmbH}$ ), according to the manufacturer's instructions. The relative expression of lncRNA TCF7 in plasma cells was detected by reverse transcription-quantitative PCR (RT-qPCR). Moreover, patients with MM were divided into two groups, lncRNA TCF7 low expression and lncRNA TCF7 high expression, based on the median value of IncRNA TCF7 expression (2.472).

Treatment. After enrollment, all patients with MM received appropriate treatments based on their physical condition,clinical status and willingness, according to National Comprehensive Cancer Network (NCCN) Clinical Practice Guidelines in Oncology: Multiple Myeloma (2014. V2) (18). Moreover, treatment options were not based on lncRNA expression. For patients with MM, induction chemotherapy was applied, and the commonly used regimensincluded bortezomib/dexamethasone, bortezomib/doxorubicin/dexamethasone, bortezomib/thalidomide/dexamethasone, lenalidomide/low-dose dexamethasone, melphalan/prednisone/bortezomib or melphalan/prednisone/thalidomide. After 2-4 cycles of induction chemotherapy, the treatment response was assessed according to the NCCN guideline $(2014$, V2). Complete response (CR) was defined as a negative immunofixation on the serum and urine, the disappearance of any soft tissue plasmacytomas and $\leq 5 \%$ plasma cells in the bone marrow. Very good partial response (VGPR) was defined as serum and urine M-protein detectable by immunofixation, but not electrophoresis, or $\geq 90 \%$ reduction in serum M-protein plus a urine M-protein level $<100 \mathrm{mg}$ per $24 \mathrm{~h}$. Partial response (PR) was defined as 50\% reduction of serum M-protein and a reduction in $24 \mathrm{~h}$ urinary M-protein by $90 \%$ or to $<200 \mathrm{mg}$ per $24 \mathrm{~h}$. Overall response rate (ORR) was defined as the proportion of patients who achieved CR, VGPR or PR during treatment. Furthermore, the subsequent 


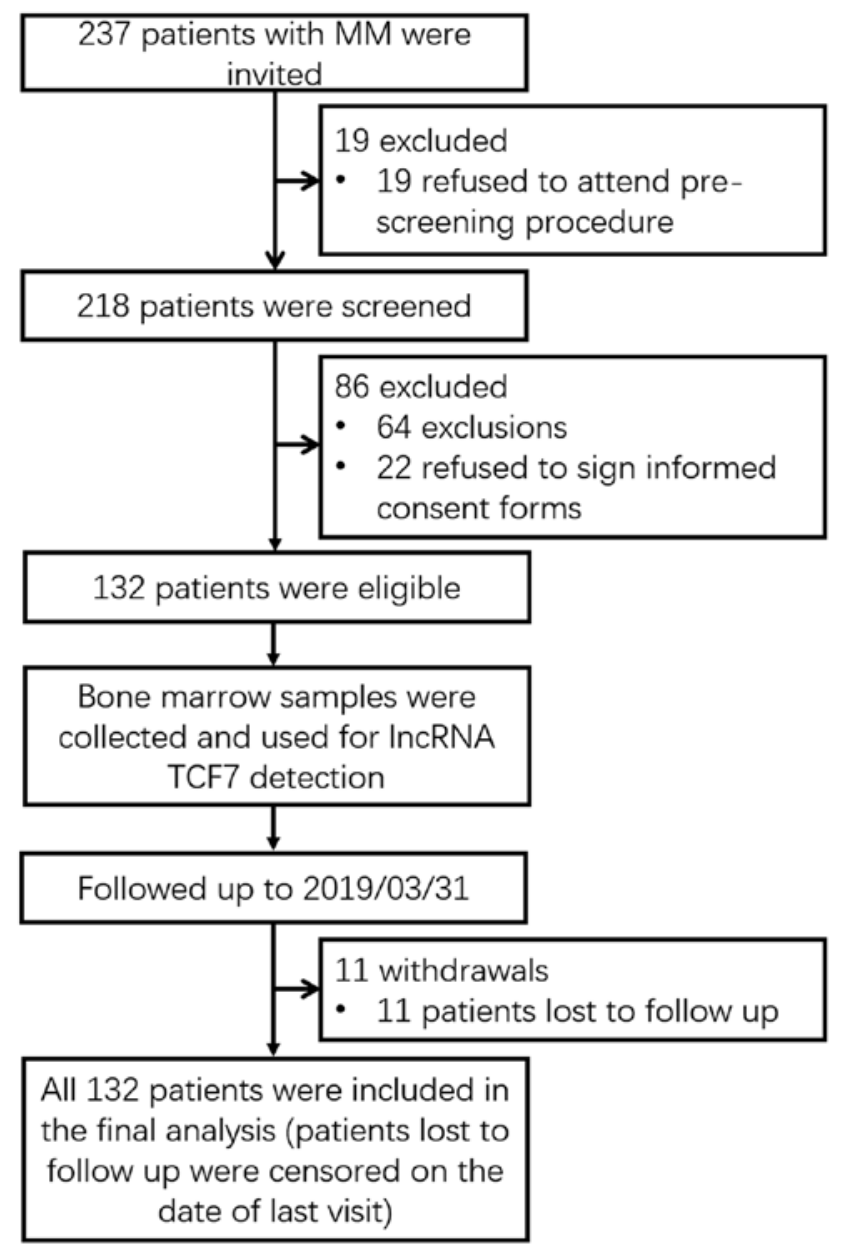

Figure 1. Study flow diagram of recruiting patients with MM and their follow-up. lncRNA TCF7, long non-coding RNA T-cell factor 7; MM, multiple myeloma.

therapies were administered as clinically indicated according to the NCCN guideline (2014. V2) (18).

Follow-up. Regular follow-up was performed by telephone or clinic visits, and the last follow-up data were collected on March 31, 2019. The median follow-up duration was 25.0 months, ranging from 2.0-36.0 months. Event-free survival (EFS) was measured from the date of entry into the study to the date of induction treatment failure, relapse from CR or mortality from any cause, and patients not known to have any of these events were censored on the date they were last examined. Overall survival (OS) was measured from the date of entry into the study to the date of mortality from any cause, and patients not known to have died at last follow-up were censored on the date they were last known to be alive.

Cell culture. The human MM cell lines OPM-2, RPMI-8226, U-266 and MOLP-2 (Leibniz Institute DSMZ-German Collection of Microorganisms and Cell Cultures $\mathrm{GmbH}$ ) were cultured in 90\% RPMI 1640 medium (Gibco; Thermo Fisher Scientific, Inc.) with 10\% FBS (Gibco; Thermo Fisher Scientific, Inc.). The human MM cell line LP-1 (Leibniz Institute DSMZ-German Collection of Microorganisms and Cell Cultures $\mathrm{GmbH}$ ) was cultured in $90 \%$ Iscove's Modified
Dulbecco's Medium(Gibco; ThermoFisher Scientific,Inc.) with 10\% FBS (Gibco; Thermo Fisher Scientific, Inc.). The human fetal osteoblastic cell line hFOB1.19 (American Type Culture Collection) was cultured in 90\% DMEM (Sigma-Aldrich; Merck KGaA) with 10\% FBS (GE Healthcare). All cell lines were cultured in a humidified atmosphere of $95 \%$ air and $5 \% \mathrm{CO}_{2}$ at $37^{\circ} \mathrm{C}$.

lncRNA TCF7 detection in cell lines. The relative expression of lncRNA TCF7 in human MM cell lines was detected by RT-qPCR, and human fetal osteoblastic cell line hFOB1.19 was used as a control.

Transfection and detections. The lncRNA TCF7 overexpression (OE) plasmid (ID:NR_033449.2 full length) and lncRNA TCF7 knockdown (KD) plasmid (using a short hairpin RNA, 5'-CACCGCACCTAGGTGCTCACTGAA CGAATTCAGTGAGCACCTAGGTG-3') were constructed by Shanghai Qeejen Bio-Tech Co., Ltd. with pEX-2 vector and pGPH1 vector (both BioVector NTCC, Inc.), respectively. PEX-2 vector cloned with a non-sense DNA fragment (5'-AACACCGAACGAGACAGGATT-3') was constructed as the control OE plasmid, and pGPH1 vector cloned with a non-sense DNA fragment (5'-CACCGTTCTCCGAACGTG TCACGTCGAAACGTGACACGTTCGGAGAA-3') was constructed as the control KD plasmid. Subsequently, HilyMax (Dojindo Molecular Technologies, Inc.) was used to transfect $0.8 \mu \mathrm{g}$ control OE plasmid, IncRNA TCF7 OE plasmid, control KD plasmid and lncRNA TCF7 KD plasmid into RPMI-8226 and U-266 cells ( $1 \times 10^{6}$ cells/well) at $37^{\circ} \mathrm{C}$ for $6 \mathrm{~h}$. Cells transfected with control OE plasmid were defined as the OE-NC group, those transfected with lncRNA TCF7 OE plasmid were defined as the OE-TCF7 group, those transfected with control $\mathrm{KD}$ plasmid were defined as the KD-NC group and those transfected with lncRNA TCF7 KD plasmid were defined as the KD-TCF7 group.

After $24 \mathrm{~h}$ of transfection, the relative expression levels of lncRNA TCF7 in RPMI-8226 and U-266 cells were detected using RT-qPCR. Cell proliferation at 0, 24, 48 and $72 \mathrm{~h}$ post-transfection was detected using the Cell Counting Kit-8 (CCK-8), and the apoptotic rate was detected by Annexin V (AV)/propidium iodide (PI) assay at $48 \mathrm{~h}$ post-transfection. Experiments were conducted three times to optimize the experimental condition, and to ensure that transfection with lncRNA TCF7 OE and KD plasmids was successful.

Target gene detection. MicroRNA (miRNA/miR)-200c has been reported to be a direct target gene of lncRNA TCF7 in cancer types other than MM (11), and miR-200c may act as a tumor suppressor in several hematological malignancies, such as acute lymphoblastic leukemia (19). Therefore, it was hypothesized that lncRNA TCF7 may function via miR-200c in $\mathrm{MM}$; therefore, the present study detected the relative expression levels of miR-200c in OE-NC, OE-TCF7, KD-NC and KD-TCF7 groups 48 h post-transfection.

$R T$ - $q P C R$. IncRNA TCF7 and miR-200c relative expression levels in plasma cells were evaluated by RT-qPCR. Total RNA was extracted using TRIzol ${ }^{\circledR}$ reagent (Invitrogen; Thermo Fisher Scientific, Inc.). Subsequently, total RNA was 
quantified using NanoDrop 2000 (Thermo Fisher Scientific, Inc.) and cDNA was synthesized using PrimeScript RT reagent kit (Takara Bio, Inc.) at $37^{\circ} \mathrm{C}$ for $15 \mathrm{~min}$ and $85^{\circ} \mathrm{C}$ for 5 sec. qPCR was performed using TB Green Fast qPCR mix (Takara Bio, Inc.) on the ABI HT RT PCR system 7900 (Applied Biosystems; Thermo Fisher Scientific, Inc.). The PCR amplification program was performed as follows: $95^{\circ} \mathrm{C}$ for $30 \mathrm{sec}$, followed by $40 \mathrm{cycles}$ of $95^{\circ} \mathrm{C}$ for $5 \mathrm{sec}$ and $60^{\circ} \mathrm{C}$ for $30 \mathrm{sec}$. IncRNA TCF7 relative expression was calculated by $2^{-\Delta \Delta C q}$ using GAPDH as an internal reference, and miR-200c relative expression was calculated by $2^{-\Delta \Delta C q}$ using U6 as an internal reference (20). The primers used were as follows: IncRNA TCF7, forward 5'-GAAGCCCGTATTAGA CTGAATGGT-3', reverse 5'-TTGAGACAATCTGGTATG AGGACAC-3'; GAPDH, forward 5'-GACCACAGTCCATGC CATCAC-3', reverse 5'-ACGCCTGCTTCACCACCTT-3'; miR-200c, forward 5'-ACACTCCAGCTGGGCGTCTTACCC AGCAGT-3', reverse 5'-TGTCGTGGAGTCGGCAATTC-3'; and U6, forward 5'-CGCTTCGGCAGCACATATACTA-3' and reverse 5'-ATGGAACGCTTCACGAATTTGC-3'.

CCK-8 assay. RPMI-8226 and U-266 cells (both $5 \times 10^{3}$ cells/well) were seeded into a 96-well plate and incubated at $37^{\circ} \mathrm{C}$ in an atmosphere containing $95 \%$ air and $5 \%$ $\mathrm{CO}_{2}$. Subsequently, $10 \mu \mathrm{l}$ CCK-8 reagent (Sigma-Aldrich; Merck KGaA) and $100 \mu 1$ RPMI 1640 medium were added to each well and incubated at $37^{\circ} \mathrm{C}$ for $2 \mathrm{~h}$. Optical density was measured using an ELx800 microplate reader (BioTek Instruments, Inc.) to evaluate cell proliferation.

AV/PI. RPMI-8226 and U-266 cells (both $2 \times 10^{5}$ cells/well) were collected and suspended in PBS (Sigma-Aldrich; Merck $\mathrm{KGaA}$ ) at $48 \mathrm{~h}$ post-transfection. Subsequently, $5 \mu \mathrm{l} \mathrm{AV}$ and $5 \mu 1$ PI staining solution of the Annexin V-FITC Apoptosis Detection kit (Thermo Fisher Scientific, Inc.) were added to the cell suspension and incubated at $37^{\circ} \mathrm{C}$ for $15 \mathrm{~min}$ in dark. Then, flow cytometry was performed using CytoFLEX system (Beckman Coulter, Inc.) to detect early and late apoptosis, and FlowJo software 7.6 (FlowJo LLC) was used for data analysis.

Statistical analysis. Experiments were repeated three times. Continuous variables were checked for normality using the Kolmogorov-Smirnov test. Normally distributed data are presented as the mean \pm SD and data that were not normally distributed are presented as the median and interquartile range (IQR). Categorical variables were presented as count (\%). Comparisons were determined by independent-sample t-test, $\chi^{2}$ test, Mann-Whitney U test or one-way ANOVA followed by Dunnett's test. Receiver operating characteristic (ROC) curves were plotted, and the area under the curve (AUC) with 95\% CI was calculated to assess the distinguishable value of variables between subjects. EFS and OS were displayed with Kaplan-Meier curves, and the difference between groups was determined by log-rank test. Statistical analyses were performed using SPSS 24.0 (SPSS, Inc.), and figures were plotted using GraphPad Prism 7.00 (GraphPad Software, Inc.) and FlowJo Software 7.6 (FlowJo LLC). All tests were two-sided, and $\mathrm{P}<0.05$ was considered to indicate a statistically significant difference.

\section{Results}

Study flow. Initially, 237 patients with MM were enrolled, and 19 patients were excluded from the study due to refusal to attend a pre-screening procedure (Fig. 1). Thus, 218 patients were screened for eligibility; 86 patients were excluded (64 patients did not meet inclusion criteria or met exclusion criteria, and 22 patients refused to sign informed consent forms). Subsequently, bone marrow samples were collected from 132 eligible patients and used for lncRNA TCF7 detection. Eligible patients were followed-up until March 31, 2019. During the follow-up period, there were 11 withdrawals due to lost follow up. All 132 patients were included in the final analysis, and patients who were lost during follow up were censored on the date of last visit.

Baseline characteristics of patients with MM. The mean age of patients with MM was $57.2 \pm 8.2$ years, and there were $82(62.1 \%)$ male patients and 50 (37.9\%) female patients. Moreover, the number of patients with MM with Durie-Salmon stage II and III were $56(42.4 \%)$ and $76(57.6 \%)$, respectively. Regarding ISS stage, the number of patients with ISS stage I, II and III were 35 (26.5\%), 45 (34.1\%) and 52 (39.4\%), respectively. Other detailed characteristics of patients with MM are listed in Table I.

Comparison of lncRNA TCF7 expression between patients with MM and controls. The results demonstrated that lncRNA TCF7 expression was elevated in patients with MM [median (IQR), 2.472 (1.375-4.029)] compared with controls [median (IQR), 1.016 (0.440-1.727); P<0.001; Fig. 2A). Furthermore, ROC curve analysis identified that lncRNA TCF7 expression may distinguish patients with MM from controls (AUC, 0.793; 95\% CI, 0.725-0.861; Fig. 2B).

Association of IncRNA TCF7 expression with the clinical characteristics of patients with MM. Patients with MM were divided into lncRNA TCF7 low expression and lncRNA TCF7 high expression groups based on the median value of lncRNA TCF7 expression (2.472; Table I). It was revealed that lncRNA TCF7 high expression was associated with higher $32-\mathrm{MG}$ $(\mathrm{P}=0.001)$, more advanced ISS stage $(\mathrm{P}<0.001)$ and more $\mathrm{t}$ $(14 ; 16)$ mutations $(\mathrm{P}=0.011)$ in patients with MM. However, no associations of lncRNA TCF7 expression with age $(\mathrm{P}=0.184)$, sex $(\mathrm{P}=1.000)$, immunoglobulin subtype $(\mathrm{P}=0.162)$, presence of bone lesions $(\mathrm{P}=0.086), \mathrm{Hb}(\mathrm{P}=0.301)$, calcium $(\mathrm{P}=0.857)$, Scr $(\mathrm{P}=0.115)$, ALB ( $\mathrm{P}=0.601)$, LDH ( $\mathrm{P}=0.726)$, Durie-Salmon stage $(\mathrm{P}=0.159), \mathrm{t}(4 ; 14)(\mathrm{P}=0.572)$ or $\operatorname{Del}(17 \mathrm{p}) ; \mathrm{P}=0.286)$ were observed in patients with MM.

Association of IncRNA TCF7 expression with treatment response in patients with $M M$. It was demonstrated that $27.3 \%$ of patients achieved CR and $76.5 \%$ of patients achieved ORR (Fig. 3A). Moreover, patients with CR [1.781 (0.982-3.065)] presented with lower lncRNA TCF7 expression compared with patients without $\mathrm{CR}$ [2.574 (1.728-4.249); $\mathrm{P}=0.022$; Fig. 3B]. The ROC curve analysis results indicated that lncRNA TCF7 expression could differentiate patients with CR from patients without CR (AUC, 0.630; 95\% CI, 0.521-0.739; Fig. 3C). Furthermore, no difference in lncRNA TCF7 mRNA 
Table I. Characteristics of patients with multiple myeloma $(n=132)$ with high and low lncRNA TCF7 expression ( $\mathrm{n}=66$ each).

\begin{tabular}{|c|c|c|c|c|}
\hline Characteristics & Total & $\begin{array}{l}\text { lncRNA TCF7 } \\
\text { high expression }\end{array}$ & $\begin{array}{l}\text { lncRNA TCF7 } \\
\text { low expression }\end{array}$ & P-value \\
\hline Mean age $\pm S D$, years & $57.2 \pm 8.2$ & $56.2 \pm 7.9$ & $58.1 \pm 8.5$ & 0.184 \\
\hline Sex, n $(\%)$ & & & & 1.000 \\
\hline Male & $82(62.1)$ & $41(62.1)$ & $41(62.1)$ & \\
\hline Female & $50(37.9)$ & $25(37.9)$ & $25(37.9)$ & \\
\hline Immunoglobulin subtype, $\mathrm{n}(\%)$ & & & & 0.162 \\
\hline $\operatorname{IgG}$ & $73(55.3)$ & $34(51.5)$ & $39(59.1)$ & \\
\hline $\operatorname{Ig} \mathrm{A}$ & $30(22.7)$ & $12(18.2)$ & $18(27.3)$ & \\
\hline $\operatorname{IgM}$ & $2(1.5)$ & $2(3.0)$ & $0(0.0)$ & \\
\hline $\operatorname{IgD}$ & $3(2.3)$ & $2(3.0)$ & $1(1.5)$ & \\
\hline Bence-Jones protein & $24(18.2)$ & $16(24.3)$ & $8(12.1)$ & \\
\hline Bone lesion, n (\%) & $93(70.5)$ & $51(77.3)$ & $42(63.6)$ & 0.086 \\
\hline \multicolumn{5}{|l|}{ Biochemical indexes } \\
\hline Mean $\mathrm{Hb} \pm \mathrm{SD}, \mathrm{g} / \mathrm{dl}$ & $9.8 \pm 2.3$ & $10.0 \pm 2.4$ & $9.6 \pm 2.3$ & 0.301 \\
\hline Median calcium (IQR), mg/dl & $9.9(8.9-11.5)$ & $9.7(8.9-11.7)$ & $10.1(9.0-11.0)$ & 0.857 \\
\hline Median scr (IQR), mg/dl & $1.6(1.3-1.9)$ & $1.7(1.4-1.9)$ & $1.5(1.2-1.9)$ & 0.115 \\
\hline Mean ALB $\pm \mathrm{SD}, \mathrm{mg} / \mathrm{dl}$ & $3.9 \pm 0.7$ & $3.9 \pm 0.7$ & $3.9 \pm 0.7$ & 0.601 \\
\hline Median $\beta 2-\mathrm{MG}$ (IQR), mg/l & $4.5(2.4-7.6)$ & $6.1(3.6-9.5)$ & $3.2(1.6-5.5)$ & 0.001 \\
\hline Median LDH (IQR), U/l & $188.9(168.6-226.3)$ & $188.9(154.0-231.6)$ & $188.8(170.0-223.3)$ & 0.726 \\
\hline Durie-Salmon stage, n (\%) & & & & 0.159 \\
\hline II & $56(42.4)$ & $32(48.5)$ & $24(36.4)$ & \\
\hline III & $76(57.6)$ & $34(51.5)$ & $42(63.6)$ & \\
\hline ISS stage, n (\%) & & & & $<0.001$ \\
\hline I & $35(26.5)$ & $10(15.2)$ & $25(37.9)$ & \\
\hline II & $45(34.1)$ & $20(30.3)$ & $25(37.9)$ & \\
\hline III & $52(39.4)$ & $36(54.5)$ & $16(24.2)$ & \\
\hline \multicolumn{5}{|l|}{ Cytogenetics, n (\%) } \\
\hline $\mathrm{t}(4 ; 14)$ & $14(10.6)$ & $8(12.1)$ & $6(9.1)$ & 0.572 \\
\hline $\mathrm{t}(14 ; 16)$ & $18(13.6)$ & $14(21.2)$ & $4(6.1)$ & 0.011 \\
\hline $\operatorname{Del}(17 p)$ & $16(12.1)$ & $10(15.2)$ & $6(9.1)$ & 0.286 \\
\hline
\end{tabular}

Comparisons were determined by independent-sample t-test, $\chi^{2}$ test or Mann-Whitney U test. $\beta 2-\mathrm{MG}, \beta 2$-microglobulin; ALB, albumin; Hb, hemoglobin; Ig, immunoglobulin; IQR, interquartile range; ISS, International Staging System; LDH, lactate dehydrogenase; lncRNA, long non-coding RNA; scr, serum creatinine.
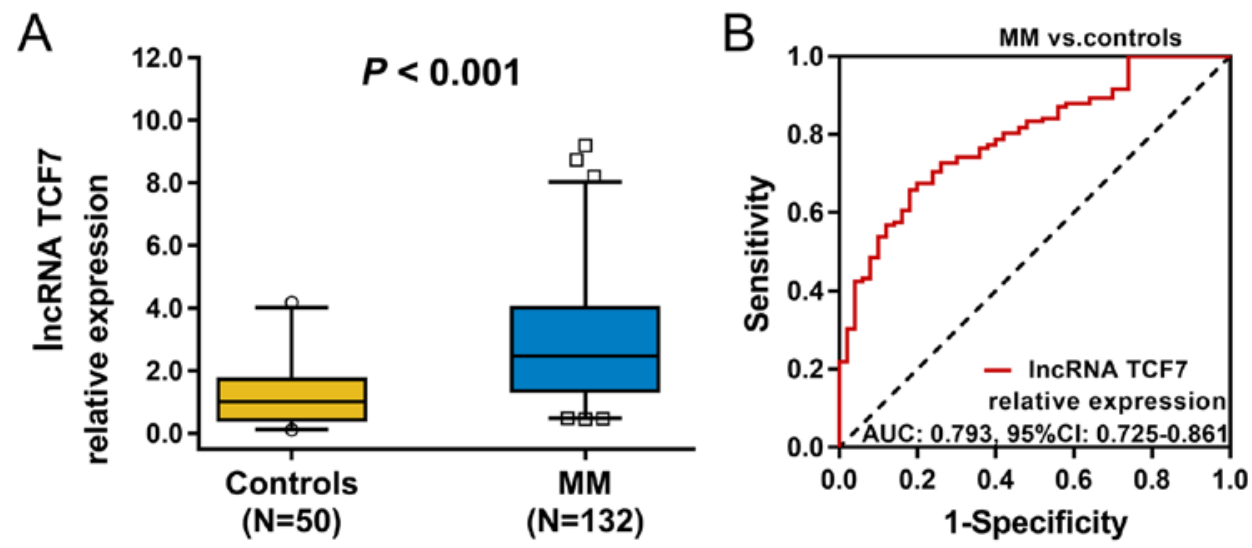

Figure 2. IncRNA TCF7 expression in patients with MM and controls. (A) Relative expression of lncRNA TCF7 in patients with MM and controls. (B) Ability of lncRNA TCF7 for predicting MM risk. Comparison between patients with MM and controls was assessed by Mann-Whitney U test. ROC curve was performed to analyze the predictive value of lncRNA TCF7 for MM risk. AUC, area under the curve; lncRNA MM, multiple myeloma; ROC, receiver operating characteristic; TCF7, long non-coding RNA T-cell factor 7. 
A

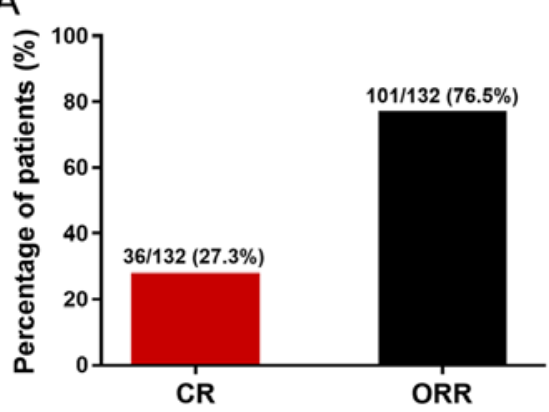

D

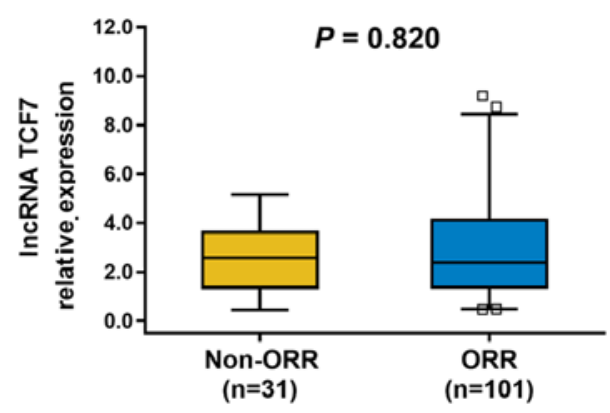

B

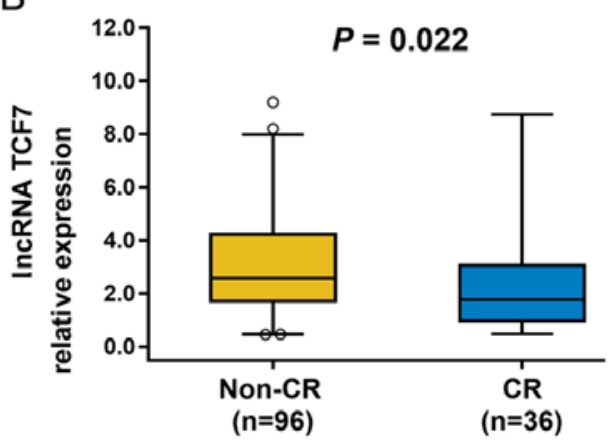

E

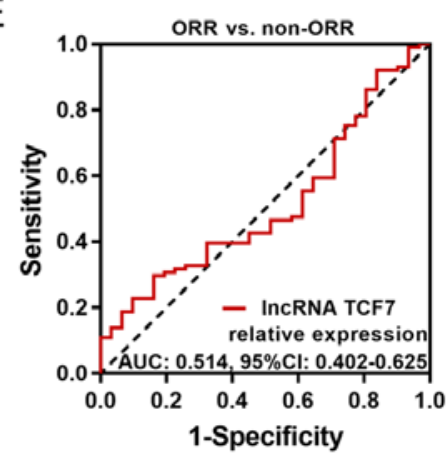

C

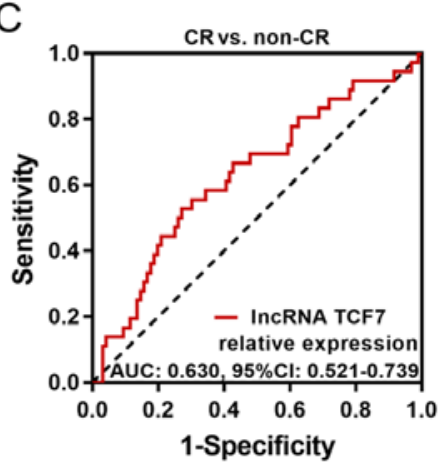

Figure 3. IncRNA TCF7 expression in patients with MM with or without treatment response. (A) Percentage of patients with CR and patients with ORR (B) Relative expression of IncRNA TCF7 in patients without CR and patients with CR. (C) Ability of lncRNA TCF7 for predicting CR. (D) Relative expression of lncRNA TCF7 in patients without ORR and patients with ORR. (E) Ability of lncRNA TCF7 in distinguishing patients without ORR and patients with ORR. Comparisons were assessed by Mann-Whitney U test. ROC curve was performed to analyze the predictive value of lncRNA TCF7 for CR and ORR in patients with MM. AUC, area under the curve; CR, complete response; lncRNA TCF7, long non-coding RNA T-cell factor 7; MM, multiple myeloma; ORR, objective response rate; ROC, receiver operating characteristic.

A

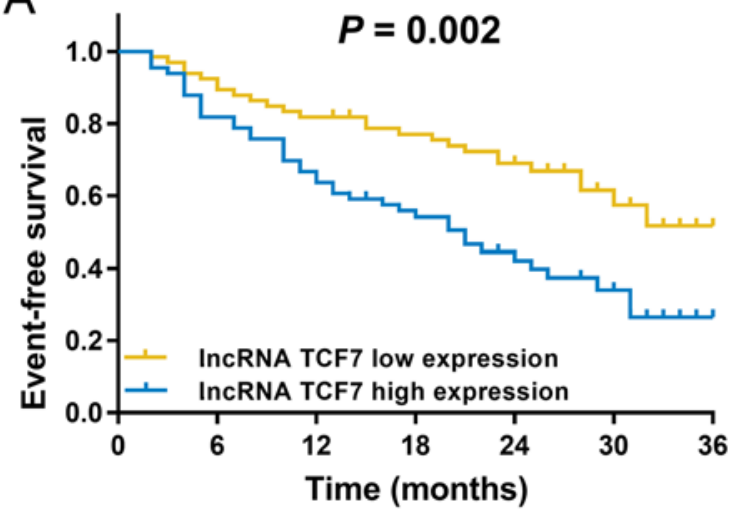

B

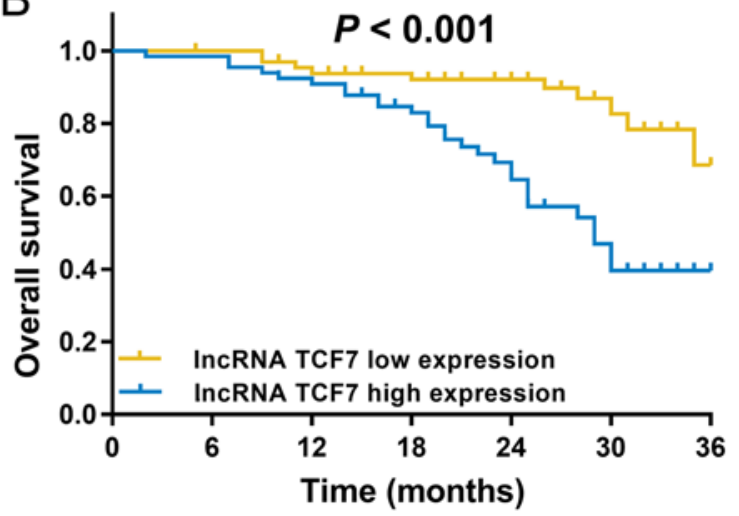

Figure 4. Association of lncRNA TCF7 relative expression with EFS and OS. (A) Association of lncRNA TCF7 expression with EFS. (B) Association of lncRNA TCF7 relative expression with OS. Kaplan-Meier curve and log-rank test were used to compare EFS and OS between patients with lncRNA TCF7 low expression and patients with lncRNA TCF7 high expression. EFS, event-free survival; lncRNA TCF7, long non-coding RNA T-cell factor 7; OS, overall survival.

expression was observed between patients with ORR and patients without ORR ( $\mathrm{P}=0.820$; Fig. 3D). Subsequent ROC curve analysis results suggested that lncRNA TCF7 expression could not distinguish patients with ORR from patients without ORR (AUC, 0.514; 95\% CI, 0.402-0.625; Fig. 3E).

Association of IncRNA TCF7 expression with survival profiles. It was demonstrated that patients with MM with lncRNA TCF7 high expression had shorter EFS compared with patients with lncRNA TCF7 low expression $(\mathrm{P}=0.002$;
Fig. 4A). Furthermore, patients with MM with high lncRNA TCF7 expression presented shorter OS compared with patients with low lncRNA TCF7 expression $(\mathrm{P}<0.001$; Fig. 4B). Therefore, the present results suggested that lncRNA TCF7 high expression was associated with poor survival profiles in patients with MM.

Comparison of lncRNA TCF7 expression between human $M M$ cell lines and a human bone marrow stromal cell line. Compared with hFOB1.19 cells, IncRNA TCF7 


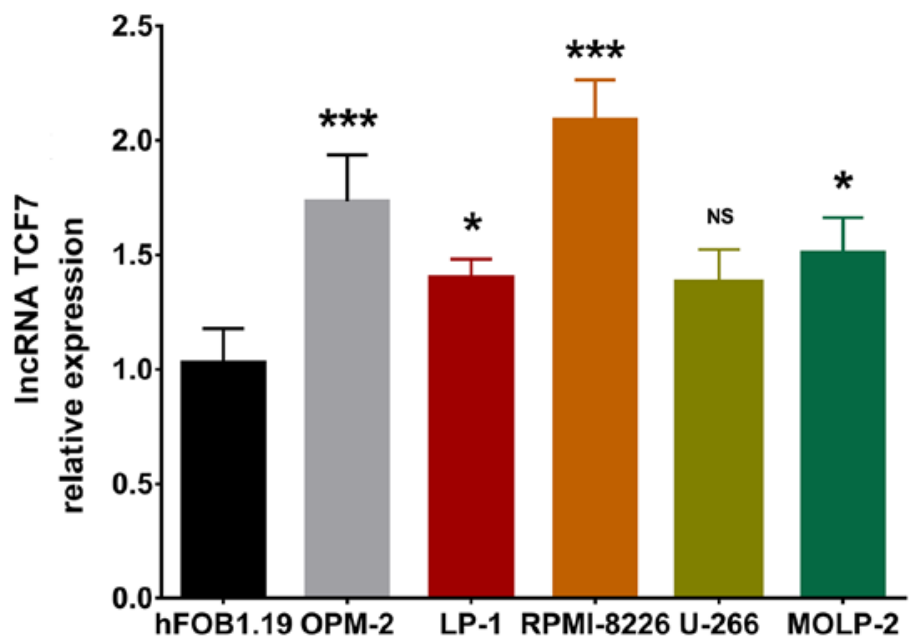

Figure 5. Expression of 1ncRNA TCF7 in hFOB1.19, OPM-2, LP-1, RPM1-8226, U-266 and MOLP-2 cells. Comparisons of 1ncRNA TCF7 relative expression between multiple myeloma cells (OPM-2, LP-1, RPM1-8226, U-266 and MOLP-2 cells) and fetal osteoblastic cells $(\mathrm{hFOB} 1.19) .{ }^{*} \mathrm{P}<0.05,{ }^{* * *} \mathrm{P}<0.001 \mathrm{vs}$. hFOB1.19. IncRNA TCF7, long non-coding RNA T-cell factor 7; NS, non-significant.

expression was upregulated in OPM-2 cells $(\mathrm{P}<0.001)$, LP-1 cells $(\mathrm{P}<0.05)$, RPMI-8226 cells $(\mathrm{P}<0.001)$ and MOLP-2 cells $(\mathrm{P}<0.05$, which were human MM cell lines (Fig. 5). However, there was no difference in lncRNA TCF7 expression between hFOB1.19 cells and U-266 cells, which is another human MM cell line. Since lncRNA TCF7 expression appeared the lowest in the U-266 cell line and the highest in the RPMI-8226 cell line among the five human MM cell lines (including OPM-2, LP-1, RPMI-8226, U-266 and MOLP-2), the effects of lncRNA TCF7 overexpression and KD were assumed to be the most efficient in U-266 and RPMI-8226 cells, respectively; therefore, these cell lines were selected for subsequent experiments.

Effects of IncRNA TCF7 on cell proliferation, apoptotic rate and $\mathrm{miR}-200 \mathrm{c}$ expression in RPMI-8226 and U-266 cells. In RPMI-8226 cells, lncRNA TCF7 expression was significantly increased in the OE-TCF7 group compared with the OE-NC group $(\mathrm{P}<0.001)$, whereas its expression was significantly lower in the KD-TCF7 group compared with the KD-NC group $(\mathrm{P}<0.001)$ at $24 \mathrm{~h}$ after transfection (Fig. 6A), thus suggesting that the transfection was successful. Furthermore, cell proliferation was increased in the OE-TCF7 group compared with the OE-NC group at $48 \mathrm{~h}(\mathrm{P}<0.05)$ and $72 \mathrm{~h}(\mathrm{P}<0.05)$, whereas it was decreased in the KD-TCF7 group compared with the KD-NC group at $48 \mathrm{~h}(\mathrm{P}<0.05)$ and $72 \mathrm{~h}(\mathrm{P}<0.01)$ post-transfection (Fig. 6B). It was also revealed that the cell apoptotic rate was inhibited in the OE-TCF7 group compared with the OE-NC group $(\mathrm{P}<0.01)$, whereas it was promoted in the KD-TCF7 group compared with the KD-NC group $(\mathrm{P}<0.01)$ at $48 \mathrm{~h}$ post-transfection (Fig. 6C and D). With regards to the potential target gene $\mathrm{miR}-200 \mathrm{c}$, the present results indicated that miR-200c expression was attenuated in the OE-TCF7 group compared with the OE-NC group $(\mathrm{P}<0.05)$. However, miR-200c expression was significantly increased in the KD-TCF7 group compared with the KD-NC group $(\mathrm{P}<0.05)$ at $48 \mathrm{~h}$ after transfection (Fig. 6E).
In U-266 cells, it was revealed that 1ncRNA TCF7 expression was significantly increased in the OE-TCF7 group compared with the OE-NC group $(\mathrm{P}<0.001)$, but it was significantly reduced in the KD-TCF7 group compared with the KD-NC group $(\mathrm{P}<0.01)$ at $24 \mathrm{~h}$ after plasmid transfection (Fig. 6F). Moreover, cell proliferation was higher in the OE-TCF7 group compared with the OE-NC group at $48 \mathrm{~h}$ $(\mathrm{P}<0.05)$ and $72 \mathrm{~h}(\mathrm{P}<0.01)$, whereas it was decreased in the KD-TCF7 group compared with the KD-NC group at $48 \mathrm{~h}$ $(\mathrm{P}<0.05)$ and $72 \mathrm{~h}(\mathrm{P}<0.05)$ post-transfection (Fig. 6G). It was demonstrated that the cell apoptotic rate was downregulated in the OE-TCF7 group compared with the OE-NC group $(\mathrm{P}<0.01)$. However, cell apoptosis was upregulated in the KD-TCF7 group compared with the KD-NC group $(\mathrm{P}<0.01)$ at $48 \mathrm{~h}$ post-transfection (Fig. $6 \mathrm{H}$ and $\mathrm{I})$. Moreover, miR-200c relative expression was significantly reduced in the OE-TCF7 group compared with the OE-NC group $(\mathrm{P}<0.05)$, whereas it was significantly increased in the KD-TCF7 group compared with the KD-NC group $(\mathrm{P}<0.05)$ at $48 \mathrm{~h}$ after transfection (Fig. 6J). Collectively, the present results suggested that lncRNA TCF7 promoted cell proliferation, but suppressed cell apoptosis and miR-200c expression in $\mathrm{MM}$.

\section{Discussion}

The present study identified the following results: i) lncRNA TCF7 expression was upregulated in patients with MM compared with controls, and lncRNA TCF7 had a good value in predicting MM risk; ii) high lncRNA TCF7 expression was associated with poor prognosis in patients with MM; and iii) lncRNA TCF7 facilitated cell proliferation, but decreased cell apoptosis and miR-200c expression in MM cells.

MM is a heterogenous malignancy of plasma cells associated with a complex pathophysiology, in which the bone marrow microenvironment and genetic abnormalities are primary contributors $(21,22)$. In the majority of patients with $\mathrm{MM}$, there is an extended period of time before the onset of symptoms, such as pain, anemia, fatigue and renal failure (23). 
A

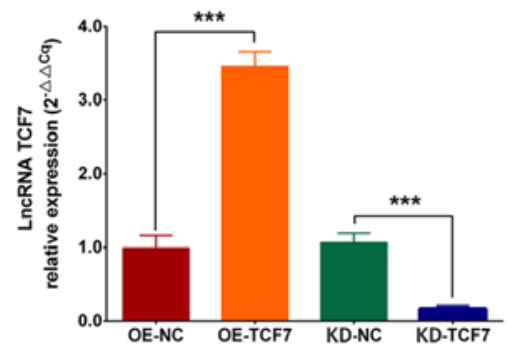

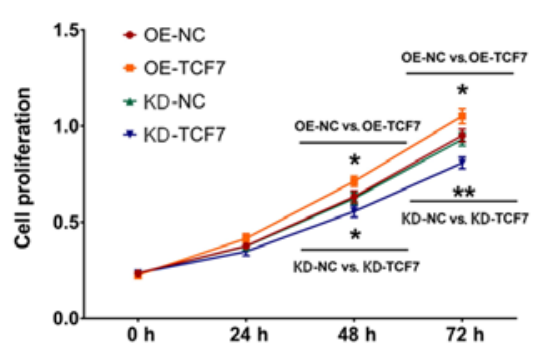

C

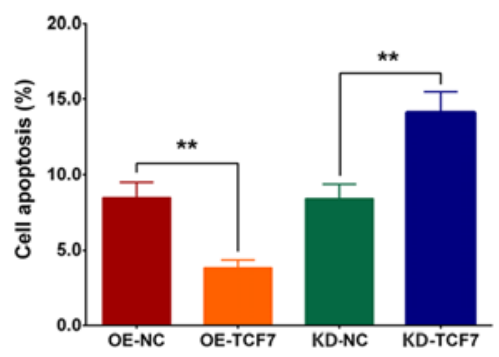

E

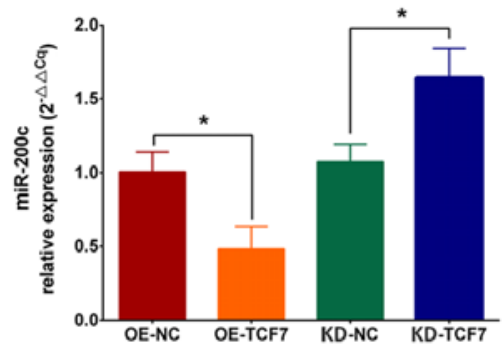

U-266 cells

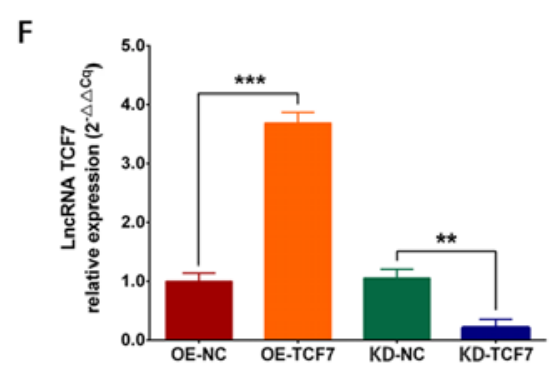

G
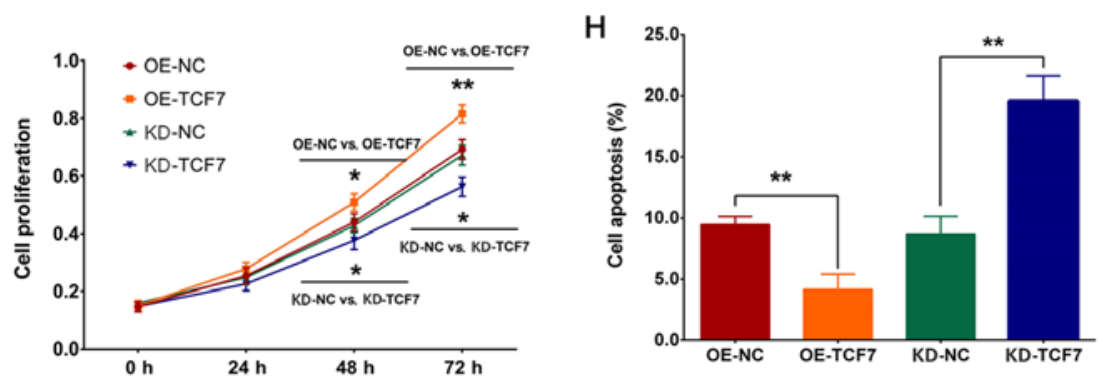

I
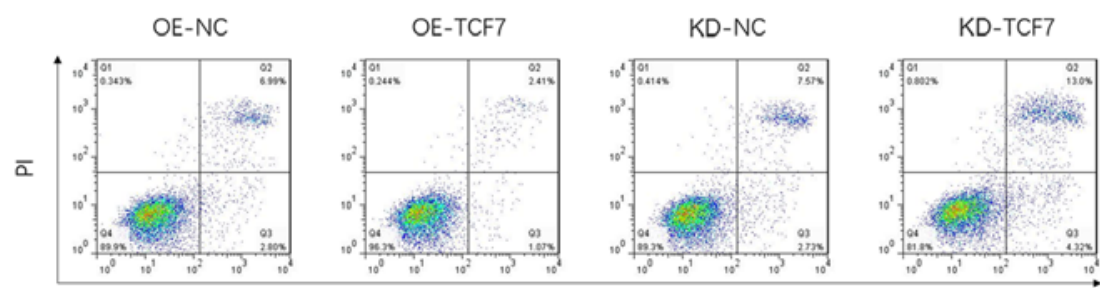

Annexin V

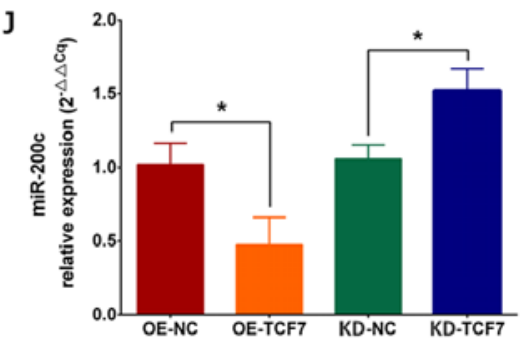

Figure 6. Influence of lncRNA TCF7 on cellular functions and miR-200c expression in RPMI-8226 and U-266 cells. (A) lncRNA TCF7 expression after transfection in RPMI-8226 cells. (B) Influence of IncRNA TCF7 on cell proliferation; (C) cell apoptosis, (D) as identified by flow cytometry; (E) and miR-200c expression in RPMI-8226 cells. (F) Expression of lncRNA TCF7 after transfection in U-266 cells. Effect of lncRNA TCF7 on (G) cell proliferation; (H) cell apoptosis, as measured by (I) flow cytometry; and (J) miR-200c expression in U-266 cells. Comparisons were determined by t-test. ${ }^{*} \mathrm{P}<0.05,{ }^{* * *} \mathrm{P}<0.01,{ }^{* * * *} \mathrm{P}<0.001$. KD, knockdown; lncRNA TCF7, long non-coding RNA T cell factor 7; miR, microRNA; NC, negative control; OE, overexpression; PI, propidium iodide.

Moreover, despite advancements in the treatment of MM, the majority of patients will eventually relapse or become refractory to these treatments $(22,24)$. Therefore, it is important to identify potential biomarkers for predicting the pathogenesis of MM.

With the wide application of high-throughput sequencing in studies investigating the potential use of lncRNAs as biomarkers, it has been revealed that lncRNAs exhibit potency as predictive biomarkers for MM risk $(25,26)$. Notably, serum IncRNA prostate cancer associated transcript 1 expression was elevated in patients with MM compared with healthy volunteers (25). Another previous study revealed that lncRNA taurine upregulated 1 expression was higher in patients with MM compared with healthy volunteers (26). With regards to lncRNA TCF7, it has been identified to trigger the activation of Wnt signaling via TCF7 expression (12). Moreover, the Wnt signaling pathway may serve a pivotal role in the development and progression of MM (13). However, the association of lncRNA TCF7 with the risk of MM is not fully understood. To the best of our knowledge, the present study was the first to 
demonstrate that lncRNA TCF7 expression was increased in patients with MM compared with controls, and that lncRNA TCF7 may be a valuable marker for differentiating patients with MM from healthy controls. This observed effect may be due to the follow reasons: i) lncRNA TCF7 may upregulate the expression of epithelial cell adhesion molecule, which enhances the self-renewal of plasma cells and results in an increased disease risk of MM (11); and ii) lncRNA TCF7 may trigger activation of the Wnt signaling pathway, which induces malignant proliferation and migration of plasma cells and leads to a higher MM risk (21). Furthermore, it has been reported that IncRNA TCF7 may activate Wnt signaling by regulating TCF7 expression (12). Thus, it was speculated that lncRNA TCF7 overexpression may promote Wnt signaling and that lncRNA TCF7 overexpression may suppress Wnt signaling. However, further experimentation is required to assess this hypothesis.

Previous studies have identified the potency of several specific lncRNAs as prognostic indicators in patients with MM (27,28). For example, patients with MM with high lncRNA colorectal neoplasia differentially expressed (CRNDE) expression had attenuated OS compared with patients with low expression of IncRNA CRNDE (27). Another study revealed that high expression of IncRNA MALAT1 was associated with shorter progression-free survival and OS in patients with MM (28). However, to the best of our knowledge, no previous studies have evaluated the association of lncRNA TCF7 with MM prognosis. The present results suggested that lncRNA TCF7 was negatively associated with EFS and OS. A reason for this effect may be due to the fact that lncRNA TCF7 was associated with increased $t(14 ; 16)$ mutations; $t(14 ; 16)$ mutation can cause overexpression of the oncogenes MAF bZIP transcription factor (MAF) and MAFB in cells, thus resulting in a more advanced stage of $\mathrm{MM}$ and poor prognosis in patients (4). Furthermore, high expression of lncRNA TCF7 was associated with higher $\beta 2-\mathrm{MG}$ level and more advanced ISS stage, and thus may indirectly lead to poor survival rates in patients with MM. Moreover, lncRNA TCF7 may induce aberrant Wnt signaling and enhance the expression of epithelial cell adhesion molecule, which increases drug resistance and the self-renewal ability of MM cells; therefore, patients with high lncRNA TCF7 expression may respond poorly to drugs and present poor prognosis (21).

IncRNAs have been proposed as competitive endogenous RNAs for miRNA, which regulate the post-transcriptional regulation of miRNAs by inhibiting their downstream activities (29). In cancer, numerous abnormally expressed lncRNAs have been suggested to facilitate malignant cell proliferation, invasion and migration $(3,8)$. Among these identified lncRNAs, lncRNA TCF7 was reported to be highly expressed in some solid cancer cells, such as colorectal cancer cells and lung cancer cells, compared with healthy cells, and it exhibited carcinogenic effects on these solid cancer types $(9,30)$. A recent study revealed that IncRNA TCF7 promoted invasion and self-renewal of tumor cells in non-small cell lung cancer (30). Moreover, another previous study demonstrated that lncRNA TCF7 facilitated the proliferation and migration of glioma cells, and that lncRNA TCF7 may directly bind to miR-200c and attenuate miR-200c expression (11). miR-200c has been proposed as a tumor suppressor that inhibits cell proliferation, migration and colony formation in MM (31). However, to the best of our knowledge, there are no previous studies examining the cellular function of lncRNA TCF7 in MM. Therefore, to further investigate the function of lncRNA TCF7 in regulating tumorigenesis of MM, the present study compared lncRNA TCF7 expression between marrow stromal cells and MM cells, and analyzed the effect of lncRNA TCF7 on the regulation of the cellular functions of two MM cell lines. The present results suggested that 1ncRNA TCF7 expression was upregulated in MM cells compared with fetal osteoblastic cells, and further cellular experiments identified that lncRNA TCF7 enhanced cell proliferation, but attenuated cell apoptosis and miR-200c expression. A possible explanation for this result could be that IncRNA TCF7 may directly bind to miR-200c, which reduces the expression of miR-200c, and subsequently facilitates the proliferation and suppresses the apoptosis of MM cells (32). However, whether lncRNA directly binds to miR-200c should be further confirmed in future studies.

In conclusion, the present results indicated that lncRNA TCF7 may be associated with higher disease risk and poor prognosis in MM. Moreover, it was demonstrated that lncRNA TCF7 enhanced cell proliferation, whereas it decreased cell apoptosis and miR-200c expression in MM cells.

\section{Acknowledgements}

Not applicable.

\section{Funding}

No funding was received.

\section{Availability of data and materials}

The datasets used and/or analyzed during the present study are available from the corresponding author on reasonable request.

\section{Authors' contributions}

TH designed the study. TD performed the experiments. RD and TH analyzed the data. TD wrote the manuscript. All authors read and approved the final manuscript.

\section{Ethics approval and consent to participate}

This study was conducted in accordance with ethical standards and was approved by the Institutional Review Board of The Central Hospital of Wuhan, Tongji Medical College, Huazhong University of Science and Technology. All participants or their guardians provided written informed consent.

\section{Patient consent for publication}

Not applicable.

\section{Competing interests}

The authors declare that they have no competing interests. 


\section{References}

1. Rollig C, Knop S and Bornhauser M: Multiple myeloma Lancet 385: 2197-2208, 2015.

2. Liu H, Xu R and Huang H: Peripheral neuropathy outcomes and efficacy of subcutaneous bortezomib when combined with thalidomide and dexamethasone in the treatment of multiple myeloma. Exp Ther Med 12: 3041-3046, 2016.

3. Sun Y, Pan J, Zhang N, Wei W, Yu S and Ai L: Knockdown of long non-coding RNA H19 inhibits multiple myeloma cell growth via NF-кB pathway. Sci Rep 7: 18079, 2017.

4. Pawlyn $\mathrm{C}$ and Morgan GJ: Evolutionary biology of high-risk multiple myeloma. Nat Rev Cancer 17: 543-556, 2017.

5. Pawlyn $\mathrm{C}$ and Davies FE: Toward personalized treatment in multiple myeloma based on molecular characteristics. Blood 133: 660-675, 2019.

6. Butova R, Vychytilova-Faltejskova P, Souckova A, Sevcikova S and Hajek R: Long non-coding RNAs in multiple myeloma. Noncoding RNA 5: 13, 2019.

7. Amodio N, Stamato MA, Juli G, Morelli E, Fulciniti M, Manzoni M, Taiana E, Agnelli L, Cantafio MEG, Romeo E, et al Drugging the lncRNA MALAT1 via LNA gapmeR ASO inhibits gene expression of proteasome subunits and triggers anti-multiple myeloma activity. Leukemia 32: 1948-1957, 2018.

8. Gu Y, Xiao X and Yang S: LncRNA MALAT1 acts as an oncogene in multiple myeloma through sponging miR-509-5p to modulate FOXP1 expression. Oncotarget 8: 101984-101993, 2017.

9. Wu B, Chen M, Gao M, Cong Y, Jiang L, Wei J and Huang J: Down-regulation of lncTCF7 inhibits cell migration and invasion in colorectal cancer via inhibiting TCF7 expression. Hum Cell 32: 31-40, 2019.

10. Jin FS, Wang HM and Song XY: Long non-coding RNA TCF7 predicts the progression and facilitates the growth and metastasis of colorectal cancer. Mol Med Rep 17: 6902-6908, 2018.

11. Zhao J, Zhang L, Zheng L, Hong Y and Zhao L: LncRNATCF7 promotes the growth and self-renewal of glioma cells via suppressing the miR-200c-EpCAM axis. Biomed Pharmacother 97: 203-208, 2018.

12. Wang Y, He L, Du Y, Zhu P, Huang G, Luo J, Yan X, Ye B, Li C, Xia P, et al: The long noncoding RNA lncTCF7 promotes self-renewal of human liver cancer stem cells through activation of Wnt signaling. Cell Stem Cell 16: 413-425, 2015.

13. Spaan I, Raymakers RA, van de Stolpe A and Peperzak V: Wnt signaling in multiple myeloma: A central player in disease with therapeutic potential. J Hematol Oncol 11: 67, 2018.

14. Ludwig H, Miguel JS, Dimopoulos MA, Palumbo A, Garcia Sanz R, Powles R, Lentzsch S, Ming Chen W, Hou J, Jurczyszyn A, et al: International myeloma working group recommendations for global myeloma care. Leukemia 28: 981-992, 2014.

15. Ross FM, Avet-Loiseau H, Ameye G, Gutiérrez NC, Liebisch P, O'Connor S, Dalva K, Fabris S, Testi AM, Jarasova M, et al: Report from the European Myeloma Network on interphase FISH in multiple myeloma and related disorders. Haematologica 97: $1272-1277,2012$

16. Durie BG and Salmon SE: A clinical staging system for multiple myeloma. Correlation of measured myeloma cell mass with presenting clinical features, response to treatment, and survival. Cancer 36: 842-854, 1975.

17. Greipp PR, San Miguel J, Durie BG, Crowley JJ, Barlogie B, Bladé J, Boccadoro M, Child JA, Avet-Loiseau H, Kyle RA, et al: International staging system for multiple myeloma. J Clin Oncol 23: 3412-3420, 2005.
18. National Comprehensive Cancer Network: NCCN Clinical Practice Guidelines in Oncology (NCCN Guidelines ${ }^{\circledR}$ ). Acute Myeloid Leukemia. Version 2. NCCN, Inc., 2014. https://www2. tri-kobe.org/nccn/guideline/hematologic/english/aml.pdf.

19. Ghodousi ES and Rahgozar S: MicroRNA-326 and microRNA-200c: Two novel biomarkers for diagnosis and prognosis of pediatric acute lymphoblastic leukemia. J Cell Biochem 119: 6024-6032, 2018.

20. Livak KJ and Schmittgen TD: Analysis of relative gene expression data using real-time quantitative PCR and the 2(-Delta Delta C(T)) method. Methods 25: 402-408, 2001.

21. van Andel H, Kocemba KA, Spaargaren M and Pals ST: Aberrant Wnt signaling in multiple myeloma: Molecular mechanisms and targeting options. Leukemia 33: 1063-1075, 2019.

22. Nagoshi H, Taki T, Hanamura I, Nitta M, Otsuki T, Nishida K, Okuda K, Sakamoto N, Kobayashi S, Yamamoto-Sugitani M, et al: Frequent PVT1 rearrangement and novel chimeric genes PVT1-NBEA and PVT1-WWOX occur in multiple myeloma with 8q24 abnormality. Cancer Res 72: 4954-4962, 2012.

23. Brigle $K$ and Rogers B: Pathobiology and diagnosis of multiple myeloma. Semin Oncol Nurs 33: 225-236, 2017.

24. Paiva B, van Dongen JJ and Orfao A: New criteria for response assessment: Role of minimal residual disease in multiple myeloma. Blood 125: 3059-3068, 2015.

25. Shen X, Zhang Y, Wu X, Guo Y, Shi W, Qi J, Cong H, Wang X, Wu X and Ju S: Upregulated lncRNA-PCAT1 is closely related to clinical diagnosis of multiple myeloma as a predictive biomarker in serum. Cancer Biomark 18: 257-263, 2017.

26. Isin M, Ozgur E, Cetin G, Erten N, Aktan M, Gezer U and Dalay N: Investigation of circulating lncRNAs in B-cell neoplasms. Clin Chim Acta 431: 255-259, 2014.

27. Meng YB, He X, Huang YF, Wu QN, Zhou YC and Hao DJ: Long noncoding RNA CRNDE promotes multiple myeloma cell growth by suppressing miR-451. Oncol Res 25: 1207-1214, 2017.

28. Handa H, Kuroda Y, Kimura K, Masuda Y, Hattori H, Alkebsi L, Matsumoto M, Kasamatsu T, Kobayashi N, Tahara KI, et al: Long non-coding RNA MALAT1 is an inducible stress response gene associated with extramedullary spread and poor prognosis of multiple myeloma. Br J Haematol 179: 449-460, 2017.

29. Shen X, Bai H, Zhu H, Yan Q, Yang Y, Yu W, Shi Q, Wang J, Li J and Chen L: Long non-coding RNA MEG3 functions as a competing endogenous RNA to regulate HOXA11 expression by sponging miR-181a in multiple myeloma. Cell Physiol Biochem 49: 87-100, 2018.

30. Wu J and Wang D: Long noncoding RNA TCF7 promotes invasiveness and self-renewal of human non-small cell lung cancer cells. Hum Cell 30: 23-29, 2017.

31. Bi C, Chung TH, Huang G, Zhou J, Yan J, Ahmann GJ, Fonseca R and Chng WJ: Genome-wide pharmacologic unmasking identifies tumor suppressive microRNAs in multiple myeloma. Oncotarget 6: 26508-26518, 2015.

32. Pour L, Sevcikova S, Greslikova H, Kupska R, Majkova P, Zahradova L, Sandecka V, Adam Z, Krejci M, Kuglik P and Hajek R: Soft-tissue extramedullary multiple myeloma prognosis is significantly worse in comparison to bone-related extramedullary relapse. Haematologica 99: 360-364, 2014.

c) (i) $\ominus$ This work is licensed under a Creative Commons Attribution-NonCommercial-NoDerivatives 4.0 International (CC BY-NC-ND 4.0) License. 